\title{
Optical confinement and colour separation in a double colour laser scanned photodiode (D/CLSP)
}

\author{
M. Vieira*, M. Fernandes, P. Louro, A. Fantoni, I. Rodrigues \\ Electronics Telecommunication and Computer Department, ISEL, Rua Conselheiro Emídio Navarro, \\ P 1949-014 Lisbon, Portugal
}

Received 1 July 2003; received in revised form 12 January 2004; accepted 20 January 2004

Available online 11 March 2004

\begin{abstract}
Large area $\mathrm{n}-\mathrm{i}-\mathrm{p}-\mathrm{n}-\mathrm{i}-\mathrm{p}$ a-SiC:H heterostructures are used as sensing element in a double colour laser scanned photodiode image sensor (D/CLSP). This work aims to clarify possible improvements, physical limits and performance of CLSP image sensor when used as non-pixel image reader. Here, the image capture device and the scanning reader are optimized and the effects of the sensor structure on the output characteristics discussed. The role of the design of the sensing element, the doped layer composition and thickness, the read-out parameters (applied voltage and scanner frequency) on the image acquisition and the colour detection process are analysed. A physical model is presented and supported by a numerical simulation of the output characteristics of the sensor.
\end{abstract}

(C) 2004 Elsevier B.V. All rights reserved.

Keywords: Tandem structures; Heterojunction; Image sensor; Electrical and numerical simulation

\section{Introduction}

Amorphous silicon-carbon $(\mathrm{a}-\mathrm{SiC}: \mathrm{H})$ is a material that exhibits excellent photosensitive properties. This feature together with the strong dependence of the maximum spectral response with the applied bias has been intensively used for the development of colour devices. Various structures and sequences have been suggested [1,2]. In our group efforts have been devoted towards the development of a new kind of colour sensor, the colour laser scanned photodiode (CLSP) [3-5]. The usual technique used for colour separation is based on the modulation of the maximum of the spectral response by the applied bias voltage [6-9] in transistor-like structures. By controlling the electrical bias of the device the effective region can be the one closer to the surface, thus sensitive to lower wavelengths, or the one deeper where only the long wavelength photons can penetrate. The bias voltages used are in the range of several volts either positive or negative.

The CLSP sensor presents a different colour separation technique, which is based on the probing of the electrical field induced across the device when illuminated with a steady-state light pattern. A low power modulated light

\footnotetext{
* Corresponding author. Tel.: +351-218317289; fax: +351-218317114. E-mail address: mv@isel.pt (M. Vieira).
}

beam, which scans the active area of the device, performs the probing by measuring the photocurrent generated by the beam, which is dependent on the local illumination conditions. When a junction is illuminated with a light pattern, a local distortion of the space charge regions occurs at the illuminated regions, giving rise to electrical field modulation across the device. Low local electrical fields are ascribed to illuminated regions and high electrical fields to dark zones.

By applying different electrical bias around the open circuit voltage the device can selectively reject different wavelengths enabling its use as a colour sensor.

A further improvement can be achieved by keeping the carriers generated by the image and by the scanner away from each other. This carrier and optical confinement can be implemented by using a double $\mathrm{p}-\mathrm{i}-\mathrm{n} / \mathrm{p}-\mathrm{i}-\mathrm{n}$ heterostructure. Advantages to this approach are high resolution, uniformity of measurement along the sensor and the cost/simplicity of the detector. The design allows a continuous sensor without the need for pixel-level patterning, and so can take advantage of the amorphous silicon technology.

This work aims to clarify possible improvements, physical limits and performance of the D/CLSP image sensor when used as non-pixel image and colour reader. Here, the image capture device and the scanning reader are optimized and the effects of the sensor structure on the output characteristics discussed. 


\section{Experimental}

Single glass $/ \mathrm{ZnO}(\mathrm{Al}) / \mathrm{p}(\mathrm{SiC}: \mathrm{H}) / \mathrm{i}(\mathrm{Si}: \mathrm{H}) / \mathrm{n}(\mathrm{SiC}: \mathrm{H}) / \mathrm{Al}$ and $\mathrm{Cr} / \mathrm{n}(\mathrm{Si}: \mathrm{H}) / \mathrm{i}(\mathrm{Si}: \mathrm{H}) / \mathrm{p}(\mathrm{Si}: \mathrm{H}) / \mathrm{n}(\mathrm{Si}: \mathrm{H}) / \mathrm{i}(\mathrm{Si}: \mathrm{H}) / \mathrm{p}(\mathrm{SiC}: \mathrm{H}) / \mathrm{Cr}$ stacked structures were produced by plasma enhanced chemical vapour deposition (PE-CVD) at $13.56 \mathrm{MHz}$ radio frequency in a three chamber load-lock UHV-system. After the deposition of the amorphous layers, a back contact of $\mathrm{Cr}$ was thermally evaporated which defines the active area of the sensor $(4 \mathrm{~cm} \times 4 \mathrm{~cm})$.

The deposition conditions of all the i-layers were kept constant while they varied in the $\mathrm{n}$ - and $\mathrm{p}$-layer by adding or not methane during the deposition process. Depending on the methane fluxes the $\mathrm{n}$ - and p-layers present conductivity and optical gaps between $1.9 \times 10^{-12}$ and $8.2 \times 10^{-7} \Omega^{-1} \mathrm{~cm}^{-1}$, 2.2 and $1.8 \mathrm{eV}$, respectively. The i-layer has a dark conductivity of $7 \times 10^{-11} \Omega^{-1} \mathrm{~cm}^{-1}$ and a photosensitivity higher than $10^{4}$ under AM1.5 illumination $\left(100 \mathrm{~mW} / \mathrm{cm}^{2}\right)$.

The characterization of the device was performed through the analysis of the photocurrent and spectral response dependencies on the applied voltage, under different optical bias conditions (dark and $\lambda_{\mathrm{L}}=450,550,650 \mathrm{~nm} ; 0<\Phi_{\mathrm{L}}<$ $450 \mu \mathrm{W} / \mathrm{cm}^{2}$ ). To suppress the dc component of the photocurrent all the measurements were performed using the lock-in technique. The responsivity was obtained by normalizing the photocurrent, $i_{\mathrm{ac}}$, to the incident flux under different light wavelengths, $\lambda_{\mathrm{S}}$ (in the range of $400-800 \mathrm{~nm}$ ), and at different optical bias, $\lambda_{\mathrm{L}}$. The collection efficiency is determined by normalizing the photocurrent to the value at which the sensor responsivity becomes independent on the optical bias (around 1.5 V).

\section{Results and discussion}

\subsection{Carrier and optical confinement}

In Fig. 1 the normalized spectral responses, $R_{\mathrm{N}}$, under different optical bias intensity, $\Phi_{\mathrm{L}}$, for: (a) a $\mathrm{p}-\mathrm{i}-\mathrm{n}$ homojunction $(\mathrm{a}-\mathrm{Si}: \mathrm{H})$ and (b) for a $\mathrm{p}-\mathrm{i}-\mathrm{n}$ heterojunction $(\mathrm{a}-\mathrm{SiC}: \mathrm{H})$ are compared. Results show that in the homojunction the spectral sensitivity is light bias independent, while in the heterojunction it depends on light bias intensity. So, the heterojunction presents light to dark sensitivity while the homojunction is blind to a pattern of light projected onto its active surface. This light bias dependence allows the use of these heterodevices as image sensors [1].

\subsection{Device description and operation}

\subsubsection{Single $p-i-n$ structure}

The CLSP operation and image representation are based on the analysis of the electrical field profile, induced across the capture device by a steady-state light pattern illumination. Low local electrical fields are ascribed to illuminated regions and high electrical fields to dark zones. In the dark regions the carriers generated by the scanner are separated by the electric field and collected, while those generated inside the illuminated regions mostly recombine inside the bulk. So, by mapping the ac component of the photocurrent, $i_{\mathrm{ac}}$, during the scanning of the capture device it is possible to reconstruct the projected light pattern.

In this device the high resistivity SiC-doped layers confine the photogenerated carriers at the different generation regions and, driven by the scanner, extract information on the image colour and intensity. In short circuit mode, it can detect a black and white image with a spatial resolution less than $20 \mu \mathrm{m}$. For reading out the RGB colour signals three forward appropriate voltages have to be successively applied in order to collect information to yield the reconstruction of a colour image.

To confirm the colour separation ability with the control of the electrical bias, one image was acquired under different electrical bias. In the image (CLSP) the first two letters were green and the last two red. Under short circuit conditions the sensor is sensitive to all wavelengths and a black/white picture is obtained (Fig. 2(a)) in this operating mode the sensor is only sensitive to the light intensity. If the bias is increased to $0.5 \mathrm{~V}$ only the green letters are observed (b) as for $0.9 \mathrm{~V}$ only the red component of the image is visible (c). The full colour picture can then be reconstructed by summing the two images (b) and (c).
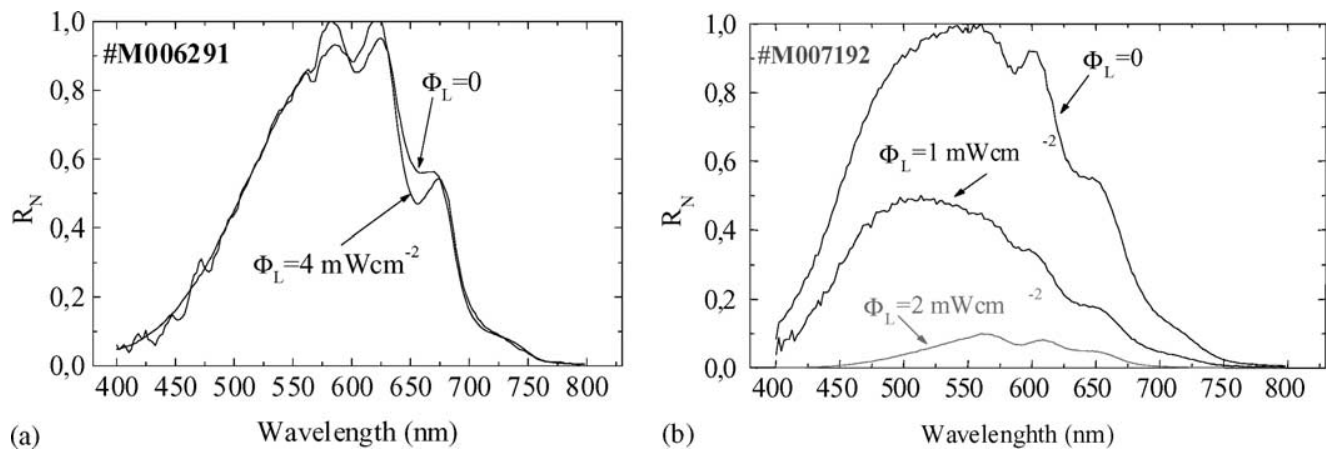

Fig. 1. Spectral response for: (a) a pin homojunction based on a-Si:H and (b) a pin heterojunction based on a-SiC:H. 


\section{CLSP CL}

(a)

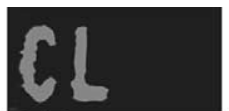

(b)

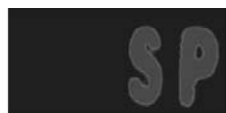

(c)
Fig. 2. Image acquired under $0 \mathrm{~V}$ (a), $0.5 \mathrm{~V}$ (b) and $0.9 \mathrm{~V}$ (c) electrical bias.

\subsubsection{Double $n-i-p-n-i-p$ structure}

When a single $\mathrm{p}-\mathrm{i}-\mathrm{n}$ heterojunction is used as sensing element, the generated carriers can diffuse away from the generated point and escape into the direction parallel to the junction. This effect leads to image smearing. The proposed carrier and optical confinement can be implemented by adding to the front photodetector (the $n-\mathrm{i}-\mathrm{p}$ homojunction) a rear thin $n-\mathrm{i}-\mathrm{p}$ a-SiC: $\mathrm{H}$ reader. In the resulting stacked structure the front a-Si:H i-layer has to be thick enough (>5000 $\AA$ ) to absorb all the light incoming from the image; the rear one is thinner $(<3000 \AA)$ and based on a-SiC:H in order to enhance light transmission from the scanner (Fig. 3).

The front photodiode, the photodetector, confines the carriers inside the illuminated regions while the rear one, the reader, driven by the optical scanner, gives information on their location (image shape), density (image intensity) and absorbed wavelength (image colour).

\subsection{Light to dark sensitivity}

Fig. 4 shows the sensor responsivity as a function of the applied voltage under different steady-state illumination conditions: (a) without optical bias (dark region, $\Phi_{\mathrm{L}}=0$ ) and (b) under red illumination (illuminated regions, $\lambda_{\mathrm{L}}=$ $650 \mathrm{~nm}, \Phi_{\mathrm{L}}=10 \mu \mathrm{W} \mathrm{cm}^{-2}$ ).

As the applied voltage increases the spectral sensitivity decreases steeply in dark and slowly under illumination. At $650 \mathrm{~nm}$ (scanner wavelength) it is much higher in dark than in the presence of optical red bias, confirming that this tandem structure can be used as an image sensor-sensing

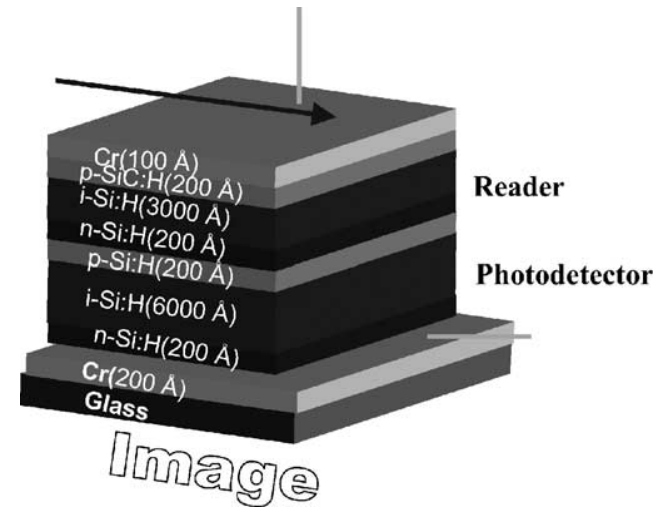

Fig. 3. D/CLSP reader and the sensing element structure.

element when a pattern of red light is projected on its active surface.

\subsection{Electrical and numerical simulation}

Both electrical and numerical simulations were performed. In Fig. 5a the simulated voltage drop across both diodes as a function of the applied voltage is displayed [3]. In Fig 5b a comparison between the experimental and simulated photocurrent are presented and in Fig. 5c the potential profile across a stacked pin-pin structure [10] is shown. Assuming that the current across the photodetector and the reader is the same and that the applied bias is shared by both, we conclude that at voltages lower then the open circuit voltage (around 1.1 V) and under illumination, the homojunction is self-forward biased and the heterojunction becomes reverse biased. The carriers generated at the illuminated diode (load, ON state) are injected into the second one (photodiode, OFF state) where they recombine, are trapped or collected, depending on its reverse current. A good fit between both experimental and simulated data was achieved.

In dark the potential drop (Fig. 5c) is distributed unevenly across both diodes. At the internal $\mathrm{p}-\mathrm{n}$ interface a rever-
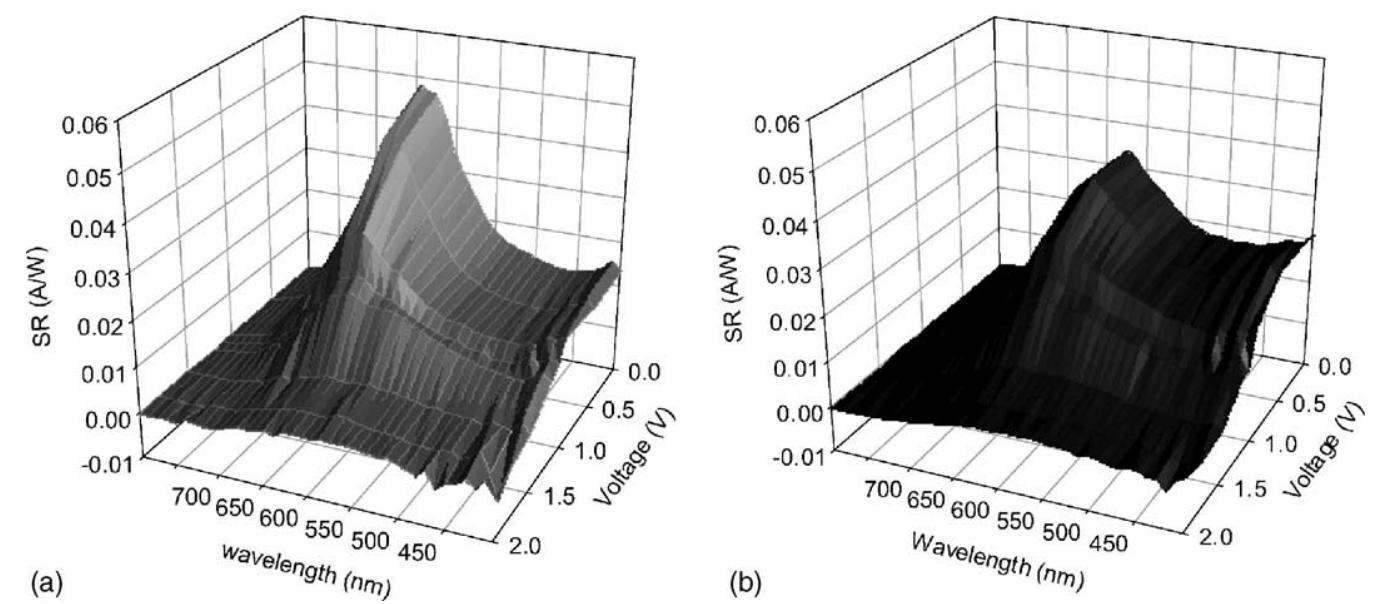

Fig. 4. Spectral response in a tandem structure under different applied bias without (a) and under red illumination (b). 

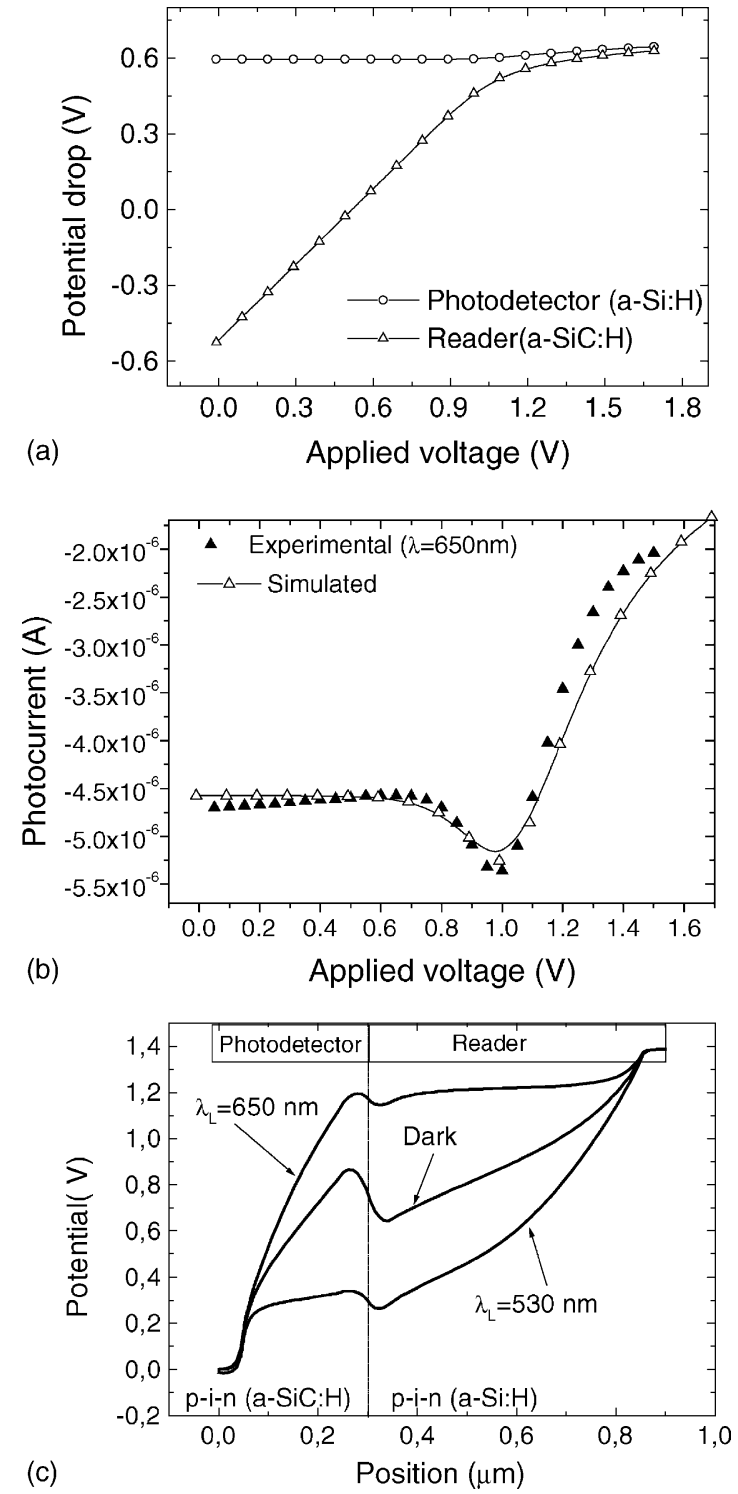

Fig. 5. (a) Voltage drop as a function of the applied bias for both homojunctions and heterojunctions. (b) Experimental and simulated photocurrent as a function of the electrical bias. (c) Potential profile across the structure.

sal in the potential profile is observed leading to charge accumulation in this region. Under illumination, and depending on the light depth penetration, a change in the potential drop across the absorber layers is observed. It flattens inside the reader under red illumination or inside the photodetector under blue/green irradiation, which ensures the presence of a quasi-neutral region inside the absorber layers. If the light is absorbed only in the front diode (blue/green light) the electrical field is always zero on the bulk of the photodetector and enhanced mainly at the reader whose i-layer becomes fully depleted. Under red illumination the light is absorbed mainly inside the reader changing the reader electrical field in an opposite way.

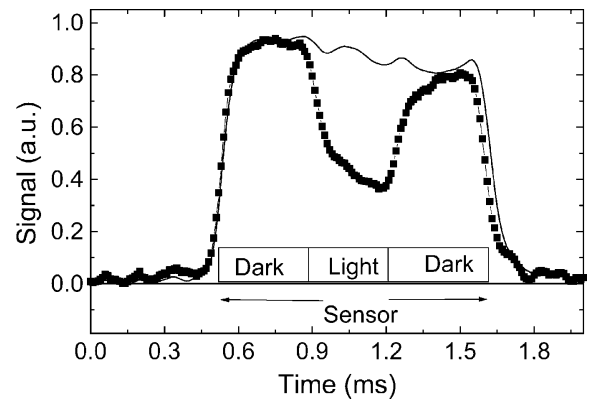

Fig. 6. Single line scans in dark (line) and crossing an illuminated region (squares).

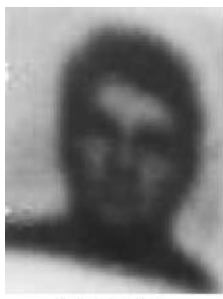

(a) CLSP

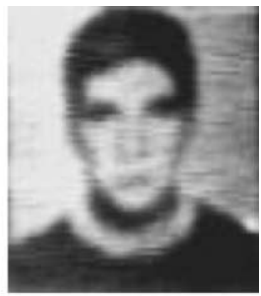

(b) D/CLSP
Fig. 7. Same image acquired using (a) single $n-i-p$ and (b) double $n-i-p-n-i-p$ structure.

\subsection{Line scanning frequency}

Fast scanning is possible with a current to voltage converter and posterior suppression of the current due to the image. Fig. 6 shows the result of a single line scan in dark (line), and crossing an illuminated area (squares). The signal represents the photocurrent due only to the scanner.

In this case the line scan frequency is close to $1 \mathrm{kHz}$ with a small reduction in the resolution caused by the value of the rise and fall times, which can be enhanced by reducing the used $1 \mathrm{k} \Omega$ load resistor. If one considers a 100 lines image then a frame rate of $10 \mathrm{~Hz}$ is obtained.

\subsection{Single versus double CLSP}

Fig. 7 compares the same image acquired using a single and a double structure showing an improved resolution. The readout frequency was also optimized leading to a value of $1 \mathrm{kHz}$, showing that scan speeds of 1000 lines per second can be achieved without a significant degradation of the resolution.

\section{Conclusions and future work}

A new design based on a stacked $n-i-p-n-i-p$ structure is proposed for the colour laser scanned photodiode sensor. Optical and carrier confinement was achieved. A B/W image was acquired under short circuit condition with improved resolution when compared with the single structure. Readout 
of 1000 lines per second was achieved allowing continuous and fast image sensing without the need of pixel patterning.

The stacked structure should be optimized in order to improve higher resolution and full colour detection. Basic image processing algorithms should be applied for image enhancement and pattern recognition. Modules for brightness calibration and edge enhancement are still needed to improve the system. Further optimisation of the optical scanning system includes dynamic characterization of the sensor (readout frequency, frame rate).

\section{Acknowledgements}

We would like to thank IPE and Univ. Waterloo for the help during this study, as well as to M. Rakhlin and Yury Vygranenko for the film deposition. This work has been financially supported by FLAD, IPL and POCTI/ESE/ $38689 / 2001$ project.

\section{References}

[1] T. Neidlinger, R. Bruggemann, H. Brummak, M.B. Schubert, Three color pin-detector using microcrystalline silicon, J. Non-Cryst. Solids 227-230 (1998) 1335-1339.

[2] D. Caputo, G. de Cesare, A switching device based on a-Si:H $\mathrm{n}-\mathrm{i}-\mathrm{p}-\mathrm{i}-\mathrm{n}$ stacked structure: modeling and characterization, IEEE Trans. Electron Dev. 43 (1996) 2109.

[3] M. Vieira, M. Fernandes, J. Martins, P. Louro, A. Maçarico, R. Schwarz, M. Schubert, Laser scanned $\mathrm{p}-\mathrm{i}-\mathrm{n}$ photodiode (LSP) for image detection, IEEE Sens. J. 1 (2) (2001) 158-167.

[4] M. Vieira, M. Fernandes, J. Martins, P. Louro, A. Maçarico, R. Schwarz, M. Schubert, Improved resolution in a P-I-N image sensor by changing the structure of the doped layers, Mat. Res. Soc. Symp. Proc. 609 (2000) A14.

[5] M. Vieira, M. Fernandes, J. Martins, P.L. Antunes, A. Macarico, R. Schwarz, M.B. Schubert, New p-i-n Si:H imager configuration for spatial resolution improvement, Sens. Actuators A 92 (2001) 60-66.

[6] C. Chang, C.-Y. Chang, Y.K. Fang, C. Jwo, The amorphous Si/SiC heterojunction color-sensitive phototransistor, IEEE Electron Dev. Lett. EDL-8 (1987) 64-65.

[7] H.-K. Tsai, S.-C. Lee, Amorphous SiC/Si three-color detector, Appl. Phys. Lett. 52 (1988) 275-277.

[8] G. De Cesare, F. Irrera, F. Lemmi, F. Palma, Amorphous Si/SiC three-color detector with adjustable threshold, Appl. Phys. Lett. 66 (1995) 1178-1180.

[9] Q. Zhu, S. Coors, B. Schneider, P. Rieve, M. Bohm, Bias sensitive a-Si(C):H multispectral detectors, IEEE Trans. Electron Dev. 45 (7) (1998).

[10] A. Fantoni, M. Fernandes, P. Louro, R. Schwarz, M. Vieira, Controlling the lateral photoeffect in a-Si:H heterojunction structures: The influence of the band offset analysed through a numerical simulation, in: Amorphous and Heterogeneous Silicon Thin Films 2001, Mat. Res. Soc. Symp. Proc. 664, 2001, p. A25.11.

\section{Biographies}

M. Vieira was born in Lisbon, Portugal in 1951. She graduated in Physics by the Faculty of Science of the University of Lisbon in 1974. In 1986, he received the Master of Science in Solid State Physics-Microelectronic by the New University of Lisbon. At that time she became Auxiliary Professor of Semiconductor and Microelectronics in ISEL, Lisbon, where she is now Coordinator Professor in Semiconductor/Electronics and President of the Department of Electronics and Communications. In 1993, she received the $\mathrm{PhD}$ in Semiconductor Materials from the New University of Lisbon. Currently, she is the head of the Group in Applied Research in Microelectronic Optoelectronic and Sensors-GIAMOS/ISEL in Lisbon, Portugal. She has several scientific papers and more than 12 years of experience in the field of thin films and devices, her research activities have been mainly related to the transient analysis and characterization of the transport properties of the semiconductor materials and device characterization.

M. Fernandes was born in Portugal in 1970. In 1993, he became researcher in the Center of Excellence for Microelectronics and Optoelectronics Processes-UNINOVA, Lisbon, Portugal. He graduated in Physics and Materials Engineering by the Faculty of Sciences and Technology from the New University of Lisbon in 1995. Currently, he is an Assistant Professor in Electronics Department of ISEL, Lisbon, Portugal, and investigator in the group of Applied Research in Microelectronic Optoelectronic and Sensors-GIAMOS in the same institution.

P. Louro was born in Portugal in 1967. In 1990, she became researcher in EID (a company of research and development in the field of electronics), Lisbon, Portugal, in the Department of Optoelectronics. She graduated in Physics by the Faculty of Sciences from the University of Lisbon in 1990. In 1995, she received the Master of Science in Material Engineering by the New University of Lisbon.

Currently, she is an Assistant Professor in the Electronics Department of ISEL, Lisbon, Portugal, where she teaches Electronic and Semiconductor Physics. Her main research interest is in the field of amorphous semiconductor thin films.

A. Fantoni was born in Rome (Italy) in 1966 . He received a university degree in Applied Mathematics from the University of Camerino, Italy (1992), and a PhD in Material Science, Micro and Optoelectronics from the New University of Lisbon, Portugal (1999). He actually teaches Semiconductor Physics in the Electronics, Telecommunications and Computer Department on the Engineering Institute of Lisbon (ISEL-DEETC). His research interests are related to numerical analysis and simulation of micro and optoelectronic thin film devices.

I. Rodrigues was born in Olhão (Portugal) in 1959. She received a university degree in Physics from the Faculty of Sciences of the University of Lisbon, Portugal (1986), and a Master's degree in Applied Mathematics from the Institute of Economics and Management of the Technical University of Lisbon, Portugal (1997). At present she teaches Mathematical Analysis in the Electronics, Telecommunications and Computer Department on the Engineering Institute of Lisbon (ISEL-DEETC) and, simultaneously she is doing her $\mathrm{PhD}$ in Sensors, Micro and Optoelectronics. Her research interests are related to numerical analysis and simulation of micro and optoelectronic devices. 